\title{
Early Ligation of the Inferior Pancreaticoduodenal Artery Using Intraoperative Ultrasonography during Pancreaticoduodenectomy
}

\author{
Takamitsu Sasaki*, Daisuke Kato, Satoshi Shinya, Kanefumi Yamashita, Ryo Nakashima, \\ Hironari Shiwaku, Yasushi Yamauchi, Tomoaki Noritomi, Yuichi Yamashita \\ Department of Gastroenterological Surgery, Fukuoka University School of Medicine, Fukuoka, Japan \\ Email: ${ }^{\text {takamitu@fc4.so-net.ne.jp }}$
}

Received 14 January 2015; accepted 5 February 2015; published 10 February 2015

Copyright (C) 2015 by authors and Scientific Research Publishing Inc.

This work is licensed under the Creative Commons Attribution International License (CC BY). http://creativecommons.org/licenses/by/4.0/

c) (i) Open Access

\begin{abstract}
Background: While the safety of pancreaticoduodenectomy (PD) has improved, the high level of difficulty associated with this operation means that the procedure carries a high mortality rate compared to other gastrointestinal operations. Various trials have been implemented in efforts to reduce the incidence of complications after PD. In particular, a large amount of intraoperative bleeding and the use of red blood cell transfusions are reportedly risk factors for postoperative complications after PD. Aim: In an attempt to reduce the amount of intraoperative bleeding during PD, consideration was given to the anatomical characteristics of the region of the pancreatic head, and the gastroduodenal artery (GDA) and inferior pancreaticoduodenal artery (IPDA) were ligated in advance of separating the head from the portal vein. We herein report the use of ultrasonography during PD to facilitate the early identification and ligation of the IPDA. Case Presentation: A 72-year-old female was diagnosed with pancreatic cancer and underwent pylorus-preserving pancreatoduodenectomy. We used ultrasonography during the operation to initially identify the IPDA and then ligate it in advance, after which the GDA was ligated before separating the pancreatic head from the superior mesenteric artery and portal vein. Identification of the IPDA was performed with the SMA as a guide using ultrasonography in Doppler mode. The amount of intraoperative bleeding was $235 \mathrm{ml}$. The patient left the hospital without any postoperative complications and has since demonstrated a good postoperative course, with no evidence of recurrent disease. Conclusions: Early ligation of the IPDA using intraoperative US is non-invasive and makes it simple to identify the IPDA. This method may be a useful technique for reducing intraoperative bleeding during the normal course of PD procedures.
\end{abstract}

\footnotetext{
${ }^{*}$ Corresponding author.
}

How to cite this paper: Sasaki, T., Kato, D., Shinya, S., Yamashita, K., Nakashima, R., Shiwaku, H., Yamauchi, Y., Noritomi, T. and Yamashita, Y. (2015) Early Ligation of the Inferior Pancreaticoduodenal Artery Using Intraoperative Ultrasonography during Pancreaticoduodenectomy. Surgical Science, 6, 50-54. http://dx.doi.org/10.4236/ss.2015.62008 


\section{Keywords}

\section{Intraoperative Bleeding, Early Ligation, Inferior Pancreaticoduodenal Artery, Pancreaticoduodenectomy, Ultrasonography}

\section{Introduction}

Pancreaticoduodenectomy (PD) has been established as a standard procedure for treating tumors in the pancreatic head region. Recently, however, while the safety of PD has improved, the difficulty of this operation means that the procedure reportedly has a relatively high mortality rate of between $1.2 \%$ and $6 \%$ [1]-[3]. Concomitant conditions that may occur subsequent to PD are reported to include, in the early stages, pancreatic and/or biliary fistula formation, gastrointestinal bleeding, intra-abdominal abscess formation, pulmonary complications and delayed gastric emptying, and, in later stages, diabetes, diarrhea and malnutrition, with between $30 \%$ and $60 \%$ of patients thought to experience these symptoms [4]-[6]. Among these complications, it has been reported that significant blood loss occurring during the operation, as well as the use of blood transfusions, is a risk factor for postoperative complications and that controlling intraoperative bleeding is therefore an extremely important issue [7]-[10]. Various trials have been implemented in efforts to reduce postoperative complications after PD. However, there are almost no reports of attempts to reduce intraoperative bleeding during PD.

The arterial blood supply of the head of the pancreas is mainly provided by the anterior and posterior pancreaticoduodenal arcades. Both arcades are formed by a superior and inferior root coming from the gastroduodenal artery (GDA) and inferior pancreaticoduodenal artery (IPDA), respectively. In regular PD procedures, only the GDA is initially ligated before separating the pancreatic head from the portal vein. According to these procedures, the blood flow in the head of the pancreas from the IPDA remains, which may result in significant blood loss at the stage at which the pancreatic head is separated from the portal vein. In one report, in an attempt to reduce intraoperative bleeding during $\mathrm{PD}$, consideration was given to the anatomical characteristics of the pancreatic head region and both the GDA and IPDA were ligated before separating the pancreatic head from the portal vein [11]-[13]. In the current study, we report the use of ultrasonography during PD to facilitate the early identification and ligation of the IPDA.

\section{Case Report}

A 72-year-old female was admitted to our hospital with a one-year history of intermittent pancreatitis. At the initial presentation, a physical examination was remarkable only for mild tenderness in the epigastrium. A computed tomography (CT) scan of the abdomen showed a small cyst in the pancreatic head. Endoscopic retrograde cholangiopancreatography (ERCP) demonstrated severe localized stenosis of the main pancreatic duct (MPD) and pooling of medium in the pancreatic head. Endoscopic ultrasonography (EUS) revealed a hypoechoic mass measuring $25 \times 15 \mathrm{~mm}$ close to the site of stenosis of the MPD. Pancreatic cancer was thus diagnosed, and pylorus-preserving pancreatoduodenectomy was conducted.

The method for performing early ligation of the IPDA using intraoperative ultrasonography was as follows: 1) the duodenum was removed at a point $3 \mathrm{~cm}$ from the pylorus ring. 2) The gallbladder was extirpated, and the duodenal ligament was dissected. 3) The pancreatic head was subjected to Kocher's maneuver, and the superior mesenteric artery (SMA) was identified. 4) Tunneling was implemented on the anterior surface of the pancreatic head and the SMA. 5) The jejunum was cut at a point approximately $10 \mathrm{~cm}$ from the ligament of Treitz. 6) The IPDA was identified using ultrasonography in Doppler mode (Figure 1(a)). It is simple to identify the SMA using ultrasonography. The SMA was therefore used as a marker to identify the IPDA running into the pancreatic uncus (Figure 1(b)), which was then ligated and removed (Figure 2). 7) Subsequent to ligature and removal of the GDA, the pancreatic head was detached from the SMA. 8) Child's reconstruction was implemented, and the PD procedure was completed. There are no differences in the technique between regular PD and our method without early ligation of the IPDA using intraoperative US.

The amount of intraoperative bleeding was $235 \mathrm{ml}$, and the patient did not receive any blood transfusions. She was discharged on the 21st postoperative day without any postoperative complications and has since demonstrated a good postoperative course, with no evidence of recurrent disease for three years after the surgery. 

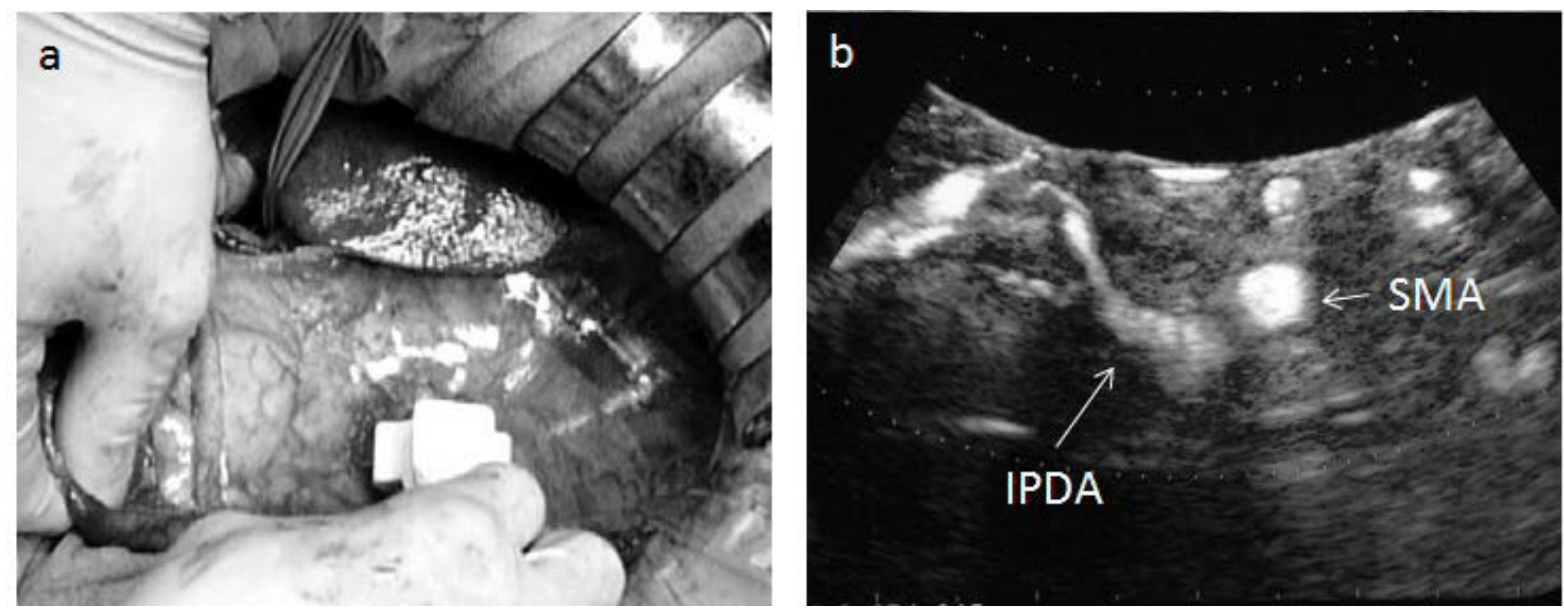

Figure 1. (a) The inferior pancreaticoduodenal artery (IPDA) was identified using intraoperative ultrasonography in Doppler mode; (b) The IPDA branched directly from the superior mesenteric artery (SMA).

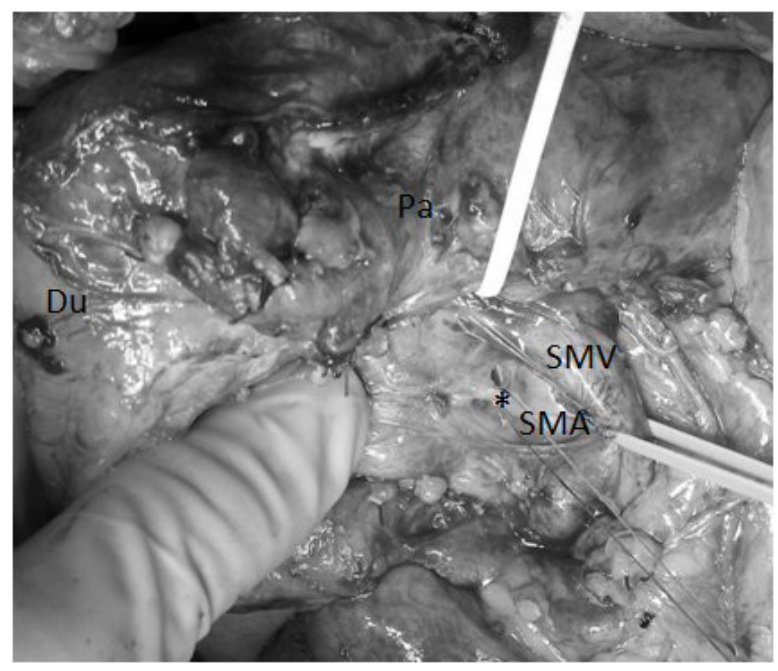

Figure 2. The IPDA, which was identified using intraoperative ultrasonography, was ligated before separating the pancreatic head from the portal vein. Du: duodenum, Pa: pancreas, SMV: superior mesenteric vein, SMA: superior mesenteric artery, asterisk: inferior pancreaticoduodenal artery.

\section{Discussion}

Compared to other types of gastrointestinal operations, PD is a procedure associated with relatively frequent complications. Significant blood loss during the operation and the use of blood transfusions are important risk factors for postoperative complications after PD [14]. The most complex operative procedure employing the PD technique, which carries the highest risk of increased blood loss, is the maneuver used to separate the pancreatic head from the superior mesenteric artery and portal vein. If the volume of blood lost during this procedure could be reduced, it may be possible to significantly decrease the volume of blood loss during the entire PD operation. In this study, we used ultrasonography to facilitate the early identification and ligation of the IPDA with the objective of reducing the amount of blood loss during PD.

The arterial blood supply of the head of the pancreas is mainly provided by the anterior and posterior pancreaticoduodenal arcades. Both arcades are formed by a superior and inferior root coming from the GDA and IPDA, respectively.

In regular PD procedures, only the GDA is initially ligated prior to pancreatectomy. Since it is not simple to identify the IPDA, in many cases, it is ligated directly prior to extirpation of the pancreatic head. In such cases, the blood flow remains, as a result of the arcade in the head of the pancreas to be removed, and the blood vessels 
that flow into the portal vein from the pancreatic head are cut, thereby causing hemostasis in the pancreatic head and thus facilitating the onset of bleeding. As a result, there is a significant amount of blood loss in the final stage of the PD procedure.

Ordinarily, the IPDA branches from the SMA, although there are often anomalies in its course. Reported techniques for early ligation of the IPDA include a method by which the IPDA is identified on CT angiography implemented prior to performing the operation (CLIP method: preoperative CT image-assessed ligation of the IPDA) [11] and/or the implementation of Kocher's maneuver, after which the IPDA is identified following further detachment along the right margin of the SMA (back-approach) [12], both of which significantly reduce intraoperative bleeding [13]. In the current case, we used ultrasonography during the operation to initially identify the IPDA and then ligate it in advance, after which the GDA was ligated before separating the pancreatic head from the superior mesenteric artery and portal vein. Identification of the IPDA was performed with the SMA as a guide using ultrasonography in Doppler mode. Subjecting the pancreatic head region to Kocher's maneuver and identifying the SMA before subjecting the portal vein to tunneling made it simple to identify the SMA using intraoperative ultrasonography. Employing the SMA as a marker, the IPDA was identified as it flowed into the pancreatic uncus. The IPDA is reported to branch via the primary jejunal artery in $57.6 \%$ of cases, directly from the SMA in $24.0 \%$ of cases and separately into the anterior IPDA and posterior IPDA in $18.4 \%$ of cases [15]-[17]. In the present case, it branched directly from the SMA.

We herein reported a case in which intraoperative ultrasonography was used to achieve early ligation of the IPDA during the normal PD procedure. Our method is non-invasive and makes it simple to identify the IPDA. This method may be useful for reducing the amount of intraoperative bleeding during the normal course of PD procedures.

\section{Conflict of Interest}

None of the authors have any conflicts of interest associated with this study.

\section{Financial Disclosure}

We have no disclosures or any financial support.

\section{References}

[1] Neoptolemos, J.P., Russell, R.C., Bramhall, S. and Theis, B. (1997) Low Mortality Following Resection for Pancreatic and Periampullary Tumours in 1026 Patients: UK Survey of Specialist Pancreatic Units. British Journal of Surgery, 84, 1370-1376. http://dx.doi.org/10.1002/bjs.1800841010

[2] Kakita, A., Yoshida, M. and Takahashi, T. (2001) History of Pancreaticojejunostomy in Pancreaticoduodenectomy: Development of a More Reliable Anastomosis Technique. Journal of Hepato-Biliary-Pancreatic Surgery, 8, 230-237. http://dx.doi.org/10.1007/s005340170022

[3] Yeo, C.J., Cameron, J.L., Sohn, T.A., Coleman, J., Sauter, P.K. and Hruban, R.H. (1999) Pancreaticoduodenectomy with or without Extended Retroperitoneal Lymphadenectomy for Periampullary Adenocarcinoma: Comparison of Morbidity and Mortality and Short-Term Outcome. Annals of Surgery, 229, 613-622.

http://dx.doi.org/10.1097/00000658-199905000-00003

[4] Conlon, K.C., Labow, D., Leung, D., Smith, A., Jarnagin, W. and Coit, D.G. (2001) Prospective Randomized Clinical Trial of the Value of Intraperitoneal Drainage after Pancreatic Resection. Annals of Surgery, 234, 487-494. http://dx.doi.org/10.1097/00000658-200110000-00008

[5] Yeo, C.J., Cameron, J.L., Sohn, T.A., Lillemoe, K.D., Pitt, H.A. and Talamini, M.A. (1997) Six Hundred Fifty Consecutive Pancreaticoduodenectomies in the 1990s: Pathology, Complications, and Outcomes. Annals of Surgery, 226, 248-260. http://dx.doi.org/10.1097/00000658-199709000-00004

[6] Cullen, J.J., Sarr, M.G. and Ilstrup, D.M. (1994) Pancreatic Anastomotic Leak after Pancreaticoduodenectomy: Incidence, Significance, and Management. The American Journal of Surgery, 168, 295-298. http://dx.doi.org/10.1016/S0002-9610(05)80151-5

[7] Miedema, B.W., Sarr, M.G., van Heerden, J.A., Nagorney, D.M., McIlrath, D.C. and Ilstrup, D. (1992) Complications Following Pancreaticoduodenectomy. Current Management. Archives of Surgery, 127, 945-949. http://dx.doi.org/10.1001/archsurg.1992.01420080079012

[8] Yeh, T.S., Jan, Y.Y., Jeng, L.B., Hwang, T.L., Wang, C.S. and Chen, S.C. (1997) Pancreaticojejunal Anastomotic Leak after Pancreaticoduodenectomy-Multivariate Analysis of Perioperative Risk Factors. Journal of Surgical Research, 
67, 119-125. http://dx.doi.org/10.1006/jsre.1996.4974

[9] Gouma, D.J., van Geenen, R.C., van Gulik, T.M., de Haan, R.J., de Wit, L.T. and Busch, O.R. (2000) Rates of Complications and Death after Pancreaticoduodenectomy: Risk Factors and the Impact of Hospital Volume. Annals of Surgery, 232, 786-795. http://dx.doi.org/10.1097/00000658-200012000-00007

[10] Kawai, M., Tani, M., Terasawa, H., Ina, S., Hirono, S. and Nishioka, R. (2006) Early Removal of Prophylactic Drains Reduces the Risk of Intra-Abdominal Infections in Patients with Pancreatic Head Resection: Prospective Study for 104 Consecutive Patients. Annals of Surgery, 244, 1-7. http://dx.doi.org/10.1097/01.sla.0000218077.14035.a6

[11] Kawai, M., Tani, M., Ina, S., Hirono, S., Nishioka, R. and Miyazawa, M. (2008) CLIP Method (Preoperative CT ImageAssessed Ligation of Inferior Pancreaticoduodenal Artery) Reduces Intraoperative Bleeding during Pancreaticoduodenectomy. World Journal of Surgery, 32, 82-87. http://dx.doi.org/10.1007/s00268-007-9305-y

[12] Ohigashi, H., Ishikawa, O., Eguchi, H., Yamada, T., Sasaki, Y. and Noura, S. (2004) Early Ligation of the Inferior Pancreaticoduodenal Artery to Reduce Blood Loss during Pancreaticoduodenectomy. Hepato-Gastroenterology, 51, 4-5.

[13] Horiguchi, A., Ishihara, S., Ito, M., Nagata, H., Shimizu, T. and Furusawa, K. (2007) Pancreatoduodenectomy in Which Dissection of the Efferent Arteries of the Head of the Pancreas Is Performed First. Journal of Hepato-BiliaryPancreatic Surgery, 14, 575-578. http://dx.doi.org/10.1007/s00534-006-1198-X

[14] Yang, Y.M., Tian, X.D., Zhuang, Y., Wang, W.M., Wan, Y.L. and Huang, Y.T. (2005) Risk Factors of Pancreatic Leakage after Pancreaticoduodenectomy. World Journal of Gastroenterology, 11, 2456-2461. http://dx.doi.org/10.3748/wjg.v11.i16.2456

[15] Bertelli, E., Di Gregorio, F., Bertelli, L. and Mosca, S. (1995) The Arterial Blood Supply of the Pancreas: A Review. I. The Superior Pancreaticoduodenal and the Anterior Superior Pancreaticoduodenal Arteries. An Anatomical and Radiological Study. Surgical and Radiologic Anatomy, 17, 97-106. http://dx.doi.org/10.1007/BF01627566

[16] Bertelli, E., Di Gregorio, F., Bertelli, L., Civeli, L. and Mosca, S. (1996) The Arterial Blood Supply of the Pancreas: A Review. II. The Posterior Superior Pancreaticoduodenal Artery. An Anatomical and Radiological Study. Surgical and Radiologic Anatomy, 18, 1-9. http://dx.doi.org/10.1007/BF03207753

[17] Bertelli, E., Di Gregorio, F., Bertelli, L., Orazioli, D. and Bastianini, A. (1997) The Arterial Blood Supply of the Pancreas: A Review. IV. The Anterior Inferior and Posterior Pancreaticoduodenal aa., and Minor Sources of Blood Supply for the Head of the Pancreas. An Anatomical Review and Radiologic Study. Surgical and Radiologic Anatomy, 19, 203-212. 
Scientific Research Publishing (SCIRP) is one of the largest Open Access journal publishers. It is currently publishing more than 200 open access, online, peer-reviewed journals covering a wide range of academic disciplines. SCIRP serves the worldwide academic communities and contributes to the progress and application of science with its publication.

Other selected journals from SCIRP are listed as below. Submit your manuscript to us via either submit@scirp.org or Online Submission Portal.
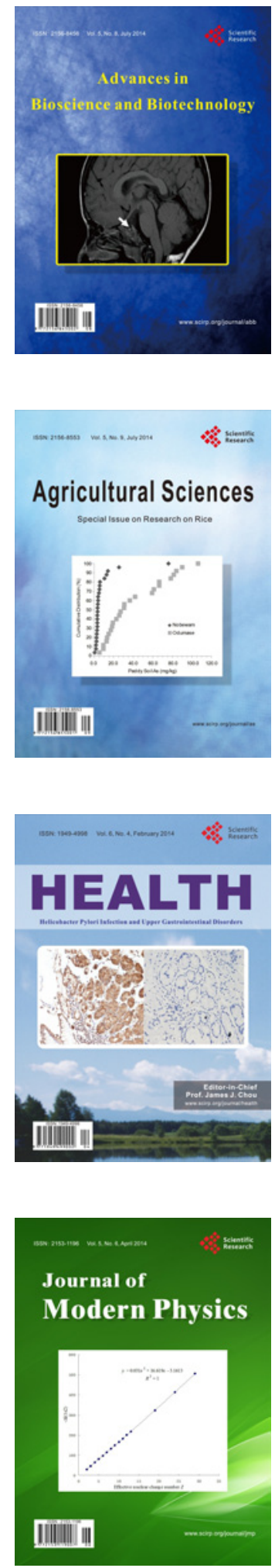
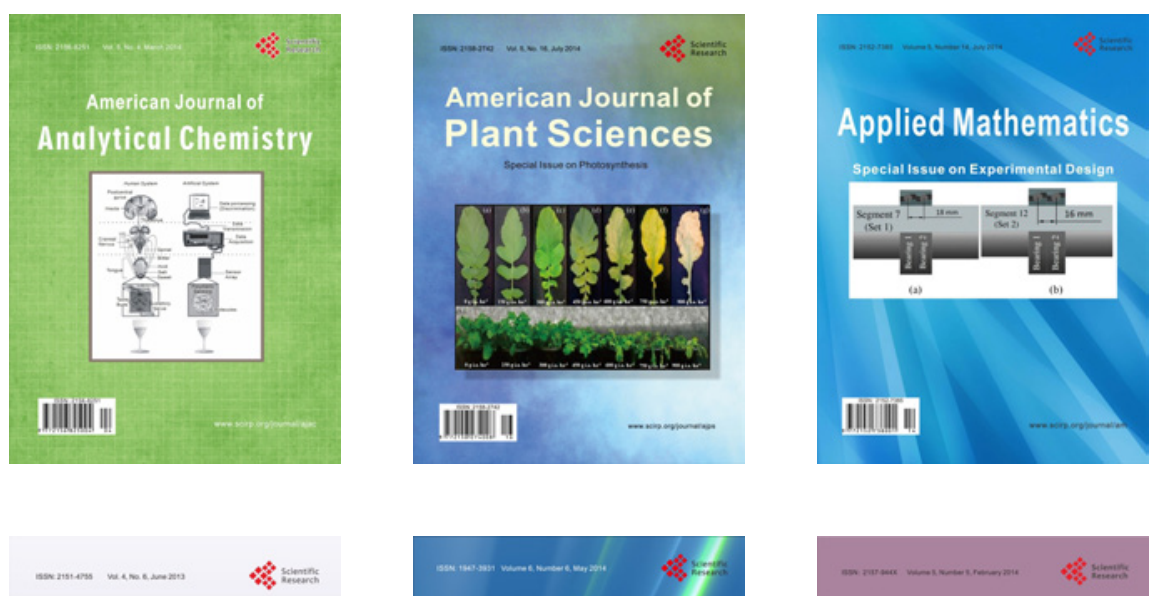

Creative Education
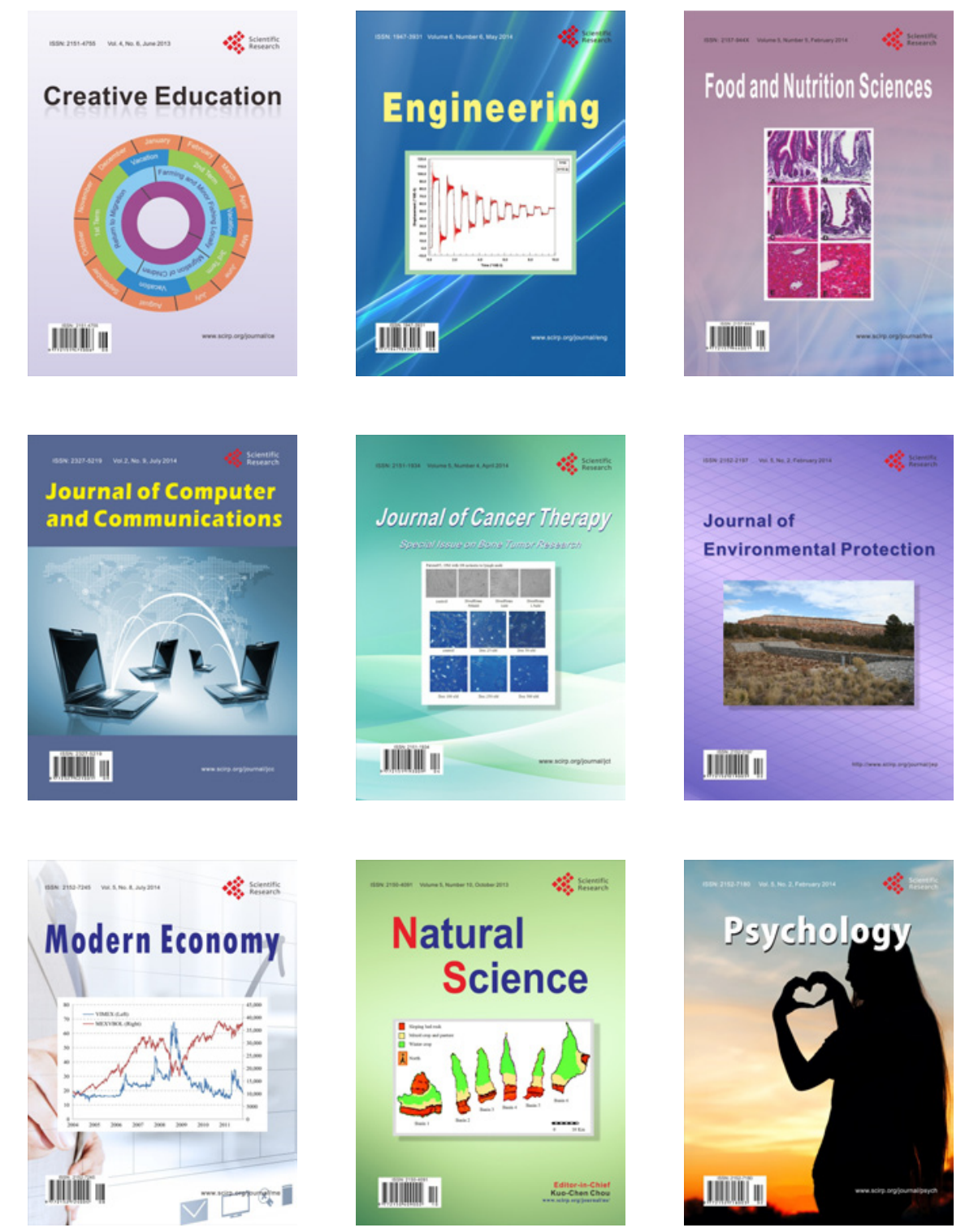